\title{
Type 2 Diabetes Mellitus in Children and Adolescents: Early Prevention and Non-Drug Therapy
}

\author{
Lunan Zhao', Ting Long², Amy Leung Hui ${ }^{3}$, Ruozhi Zhao ${ }^{3 *}$, Shaohua Long4, Wen Peng ${ }^{*}$ \\ ${ }^{1}$ Department of Physiology, McGill University, Montreal, Quebec, Canada \\ ${ }^{2}$ Department of Otolaryngology, Beijing Children's Hospital, Capital Medical University, Beijing, China \\ ${ }^{3}$ Department of Internal Medicine, University of Manitoba, Winnipeg, Canada \\ ${ }^{4}$ Xiangya Pingkuang Cooperation Hospital, Pingxiang, China \\ ${ }^{5}$ Department of Paediatrics, Xiangya Pingkuang Cooperation Hospital, Pingxiang, China \\ Email: *pengwen5706@sina.com, *ruozhi.zhao@umanitoba.ca
}

How to cite this paper: Zhao, L., Long, T., Hui, A.L., Zhao, R., Long, S.H. and Peng, W. (2017) Type 2 Diabetes Mellitus in Children and Adolescents: Early Prevention and Non-Drug Therapy. Journal of Diabetes Mellitus, 7, 121-141.

https://doi.org/10.4236/jdm.2017.73010

Received: June 2, 2017

Accepted: July 28, 2017

Published: July 31, 2017

Copyright (C) 2017 by authors and Scientific Research Publishing Inc. This work is licensed under the Creative Commons Attribution International License (CC BY 4.0).

http://creativecommons.org/licenses/by/4.0/

\begin{abstract}
The global rate of type 2 diabetes mellitus (T2DM) in youth has increased dramatically in the last 30 years. This increase mirrors the global epidemic of childhood obesity. Studies show that, compared to adults who develop T2DM, youth with T2DM ultimately suffer from more harmful symptoms. The prevalence of T2DM and obesity in youth signals a significant public health issue that financially burdens governments, families, and individuals. Since evidence suggests that T2DM in youth is different from both type 1 and type 2 diabetes in adults, researchers and clinicians face many difficulties in developing new treatments. Most treatment efforts have relied on drugs; however, recent studies suggest that non-drug therapy also effectively reduces obesity and diabetic symptoms. Healthier eating, increased physical exercise, and positive mental health, are often underappreciated factors towards managing obesity. Yet these lifestyle changes empower both young and older patients to independently fight diseases and attain better health. To manage the global health risk of obesity, further research addressing the prevention and nondrug early intervention of T2DM and obesity in youth is urgently needed. The present review focuses on the latest updates in the field.
\end{abstract}

\section{Keywords}

Diabetes, Youth, Prevention, Non-Drug Therapy

\section{Introduction}

Over the past three decades, the world-wide rate of diabetes has increased from 
108 million (4.7\% of adults), in 1980, to a startling 422 million (8.5\%), in 2014 [1]. Differing from other forms, type 2 diabetes mellitus (T2DM), also known as non-insulin dependent diabetes, occurs due to insulin resistance and deficiency, without auto-immune $\beta$-cell loss [2]. Environmental and behavioral factors help activate individual genetic predispositions towards developing T2DM. This combination of lifestyle and genetic factors leads to hepatic and muscle insulin resistance, subsequently leading to $\beta$-cell failure [3]. Many patients with T2DM are often unaware for a long time as they advance through pre-diabetic elevated glucose levels [2].

Three decades ago, T2DM was viewed as a chronic medical condition only for adults. However, since the 1990s, researchers have begun to notice a world-wide rise of childhood T2DM [4]. Currently, the Center for Disease Control and Prevention estimates that in the coming 40 years the rate of T2DM in children and adolescents will likely rise to be four times the present percentage [5]. T2DM disproportionately impacts youth of disadvantaged backgrounds as well as those from ethnic and racial minorities. Due to varying psychosocial and cultural environments, patients face many difficulties adhering to medical recommendations for lifestyle changes [6] [7]. As a result, the occurrence of both obesity and T2DM in youth continues to increase around the world [8] [9].

The presenting symptoms of T2DM and T1DM are similar and both include polyuria, polydipsia, and polyphagia. While weight loss may be present in both types of DM, obesity is the hallmark of T2DM. Upon diagnosis with T2DM, more than $85 \%$ of children were considered overweight or obese. Another easily identifiable clinical indicator of insulin resistance and T2DM is Acanthosis nigricans, a darkened, thick, velvety formation of the skin found usually in folds or creases, which is present in $90 \%$ of T2DM patients [10]. The chronic complications of T2DM include heightened risk of cardiovascular diseases, renal diseases, losses of visual acuity, and amputations, resulting in increased mortality of individuals with T2DM. In comparison to peers, adolescents diagnosed with T2DM lose, on average, fifteen years of life [11]. The earlier diagnosis of T2DM in youth both threatens their health, and, as these youth grow into adults, also potentially poses a huge socio-economic burden on the society at large. T2DM in youth is thus a significant public health issue that warrants effective prevention and early intervention.

\section{Epidemiology of T2DM in Youth}

The global rate of T2DM was once low compared to T1DM. Recent data suggests an increase in the prevalence of T2DM in several parts of the world, with regional differences. A report published by the World Health Organization (WHO) stated that the three countries with the most T2DM patients are India (with 31.7 million in 2000 and 79.4 million in 2030), China (20.8 million in 2000 and 42.3 million in 2030) and the USA (17.7 million in 2000 and 30.3 million in 2030) [12]. Specifically, the rates of T2DM in youth in the USA are nearly 12:100,000 [8] [13]. In China, the incidence of childhood T2DM rose from 
$4.1 / 100,000$ to $10.0 / 100,000$ in the past 5 years [14].

Childhood T2DM is still relatively rare in Europe (approximately 2.5:100,000), Australia (approximately 2.0:100,000), and Japan (approximately 3.0:100,000) [15] [16] [17] [18]. Rates of T2DM are higher for racial and ethnic minorities. American indigenous youth have the greatest percentage. Claims range from 0.5 cases per 1000 youth among First Nations in Manitoba to over 53 cases per 1000 youth among Pima Indians in Arizona [19] [20]. In the USA, a 2009 report found that the prevalence of T2DM for minority youth, ages 10 - 19, was 1.20 per 1000 cases for American Indian youth, 1.06 per 1000 cases for black youth, 0.79 per 1000 cases for Hispanic youth, and 0.17 per 1000 cases for white youth [21]. In Canada, the incidence of T2DM was higher in South Asian youth, ages 5 - 19, (22.88 per 100,000 patients) than in white youth (17.48) or Chinese youth (11.47) [22].

The occurrences of T2DM onset rise with patient age. The SEARCH for Diabetes in Youth study found that while the rate of T2DM for children ages 0 to 9 years was 1 per 100,000 youth, for children ages 10 to 19 , it rose to 42 per 100,000 youth [23]. The peak age of T2DM onset in children coincides with pubertal timing since the mean age at diagnosis is 12 to 16 years [10]. T2DM rates were also higher among females than among males, likely because girls are more insulin-resistant and have more subcutaneous fat than boys [21] [24].

Family history of T2DM positively correlates to chances of developing the condition, since $75 \%$ - $100 \%$ of youth patients have a first- or second-degree relative with T2DM [24] [25]. Epidemiologic studies report that children of mothers who have diabetes face greater probabilities of developing T2DM, though breastfeeding appears to have protective effects against T2DM development [26]. For both obesity and T2DM rates of youth, there are patterns of economic and ethnic disparities. Ethnic minority or low-income children are more likely to be obese than white or middle-class youth [27]. An economic study in China found that well-developed regions had a higher prevalence of T2DM compared to less-developed regions (15.16 vs. 1.64 per 100,000 persons) [14].

\section{Genetic Etiology}

Both genetic and surrounding factors increase risks for T2DM, resulting in a heterogenous etiology [28]. Since genetic risk factors are irreversible and present from birth, they cannot be modified or avoided [29]. Whereas T2DM may be genetically linked, evident by the high concordance rate of T2DM among monozygotic twins [30] and the finding that patients frequently have family members with T2DM, T1DM shows less evidence of genetic transmission, for only $5 \%$ of patients with T1DM have a family member with T1DM [10] [31]. Recent reports have located multiple genetic loci that signal high risks of T2DM. Polymorphisms of the TCF7L2 gene interferes with insulin secretion and processing, leading to a 1.4 fold risk of developing T2DM [30]. Previous findings suggest that TCF7L2 and MTNR1B genes may also be involved with the development of T2DM [32]. As genetic markers of T2DM, three established loci (HMGA2, 
KCNQ1, and TCF7L2) and two novel loci (INS-IGF2, HLA-B) were identified at genome-wide significance [33]. As genetic testing advances, more genetic markers are being discovered. Yet since environmental factors, such as diet and exercise, are more modifiable than genetic factors, lifestyle treatments may help prevent the onset, as well as the development, of T2DM [29]. Thus, although there is a strong genetic basis for the condition, suggested through instances of specific genes positively correlating to the development of diabetes, environmental factors remain important to consider.

\section{Lifestyle and Genetic Factors Affecting T2DM Pathophysiology}

In both the fasting and fed conditions, glucose concentrations modulate within defined physiological values. Several processes help regulate glucose metabolism, including the sensing of glucose concentrations, the synthesis/secretion of insulin, the suppression of hepatic glucose output, and the insulin-stimulated intake of glucose in liver, intestinal, and skeletal muscle. Insulin secretion and insulin sensitivity regulate the balance of physiological glucose levels [34]. Derangements in any of these processes, including defects during insulin receptor function, during the insulin receptor-signal transduction pathway, during glucose transport and phosphorylation, during glycogen synthesis, or during glucose oxidation, can all contribute to muscle insulin resistance and lead to hyperglycemia [35]. T2DM is hard to clinically discern from type 1 diabetes mellitus (T1DM) in the youth population. Typically, T1DM results from immune-mediated destruction of $\beta$-cells, leading to insulinopenia. In contrast, T2DM, previously known as non-insulin dependent diabetes, results from obesity-mediated insulin resistance and deficiency without auto-immune $\beta$-cell loss [2].

Lifestyle and circumstantial conditions may augment genetic susceptibility to T2DM [36]. The disease's core metabolic defect is insulin resistance, of varying degrees, which leads to subsequently progressive insulinopenia. Obesity is related to T2DM, since expanding adipose tissue, which develops in obese patients, synthesizes and secretes signaling proteins, such as leptin and tumor necrosis factor-alpha (TNF $\alpha$ ). These metabolites alter insulin secretion and receptor sensitivity. In both experimental and clinical conditions, they have even been found to cause insulin resistance [37]. This cycle of obesity and T2DM worsens hyperglycemia and further deranges physiological metabolism. Sedentary lifestyles, combined with high calorie diets, have resulted in the increase of obesity and T2DM around the world. Juices and sugar-sweetened beverages are a major source of these additional calories in the diet of youth. During the past three decades Americans have, on average, increased their daily caloric consumption by $300 \mathrm{kcal}$ [38] [39]. Other significant lifestyle factors include lower rates of physical exercise among youth. Reasons for less physical activities may include increased screen time watching television or playing video games. These instances mark a shift in interest from exercise to sedimentary pass times.

Physiological changes during puberty may aid T2DM development in youth 
[40]. Throughout puberty, insulin sensitivity temporarily decreases by $\sim 50 \%$ in healthy children. To compensate for the drop in sensitivity, insulin secretion rises; in some instances, hyperglycemia may thus develop [7] [41]. This insulin resistance is thought to be caused by an increase in growth hormone and sex steroid secretions [42]. Typically, after puberty, sex steroid levels stay elevated while insulin sensitivity improves. Unsurprisingly, the peak age of T2DM presentation in children coincides with the usual age of mid-puberty, during the brief period of raised insulin resistance [35] [40]. Studies have also found racial differences in childhood insulin sensitivity.

African-American children (ages 7 - 11) have significantly higher insulin levels than white children of similar ages [43], Some authors have suggested in utero effects since South Asian newborns have, on average, elevated cord leptin and insulin levels, factors which may lead to the development of diabetes [22].

\section{Clinical Symptoms of T2DM in Youth}

Obesity is a clinical signal for the onset of T2DM, as more than $85 \%$ of children with T2DM are overweight [10] [25]. Studies have found that excess weight leads to resistance towards insulin. Even patients who are not obese or overweight by standard weight evaluations may have an increased distribution of body fat in the abdominal area [44]. Typical symptoms for children with T2DM include glucosuria, lack of ketonuria, mildpolyuria, and minimal loss of weight [25] [45]. T2DM generally has a more insidious onset than T1DM. Often, children are diagnosed during a regular check-up through evidence of hyperglycaemia or glycosuria. Through clinical visit urinanalysis, 33\% of T2DM patients are detected [25] [31]. In more severe stages of T2DM, the child has polyuria, polydipsia, blurring of vision, and weight loss. In particular, up to $33 \%$ of racial minority groups have ketonuria and up to $25 \%$ have ketoacidosis [24] [25]. In rare occurrences, ketoacidosis or a hyperglycemic state may develop and lead to stupor, coma, or (in the absence of effective treatment) death [25]. With their clinical similarities, differentiating between T2DM and T1DM has become more difficult.

Classification is vital in deciding appropriate therapy for individuals, but some cannot be distinctly classified as having T1DM or T2DM until insulin requirements decline and a non-insulin-dependent survival course develops 44 . Symptoms of insulin resistance include acanthosis nigricans and polycystic ovarian syndrome (PCOS) [25]. Acanthosis nigricans, which is a darkened, thick, velvety condition of the skin, often found in folds, is existent in $90 \%$ of patients with T2DM and is an easily identifiable clinical signal of insulin resistance [10]. PCOS is also being increasingly valued as a signal of insulin resistance in youth. Adolescents with PCOS have a $40 \%$ reduction in insulin-stimulated glucose disposal, when compared to non-hyperandrogenic control subjects [25]. Lipid disorders and hypertension, which accounts for $35 \%-75 \%$ of diabetic complications, are also more common in youth with T2DM. Hypertriglyceridemia and lower rates of high-density lipoprotein cholesterol indicate T2DM dyslipidemia [25] [44]. 
There are also noticeable insulin sensitivity differences amongst youth of different racial backgrounds. African-American children, ages 7 to 11, have much higher insulin levels compared to white children of the same age [43]. More white youth $(\sim 50 \%)$ with T2DM were asymptomatic in contrast to minorities ( 33\%) [45]. Some syndromes, which lead to drastic increases in weight, such as Klinefelter Syndrome, Prader Willi Syndrome, or Alström Syndrome, are associated with $\mathrm{T} 2 \mathrm{DM}$ and mental retardation.

\section{Complications of T2DM in Youth}

Injuries to blood vessels are the major long-term complications of T2DM. The condition increases the chance of cardiovascular illnesses by two folds [46]. "Macrovascular" diseases include coronary artery disease, which accounts for $75 \%$ of deaths in diabetic patients, [47], stroke, and peripheral vascular disease. Small blood vessels are damaged particularly in the eyes, kidneys, and nerves [48]. Damage to the blood vessels of the eye's retina, known as diabetic retinopathy, leads to gradual vision loss and blindness [48]. In the kidneys, the damage is known as diabetic nephropathy, which can lead to tissue scarring, protein loss, and long-lasting kidney malfunctions. As a result, patients may need kidney transplants or dialysis [48]. Nerve damage, known as diabetic neuropathy, is the most common complication of diabetes [48]. The symptoms include numbness, tingling, altered pain sensation, and foot ulcers. Ultimately, nerve injuries could damage skin or require amputation. Proximal diabetic neuropathy also causes painful muscle damage and muscle weakness.

Youth with T2DM have a higher risk for complications in comparison to adults with T2DM or T1DM. This increased risk is not related to glycemic levels or length of the disease but instead associated with hypertension and dyslipidemia [49] [50]. For instance, in the TODAY study, $14 \%$ of patients had drastically elevated blood pressure levels, $13 \%$ had moderate increases in urine albumin, and $80 \%$ had depressed HDL cholesterol rates [51]. In another study, SEARCH, $92 \%$ of youth with T2DM had metabolic syndrome [52]. Similarly, among Canadian First Nations youth with T2DM, 13\% of youth, on average, had drastically elevated systolic blood pressures [53]. In the SEARCH study, $4 \%$ of youth with T2DM developed Retinopathia and 28\% developed microalbuminuria [52].

\section{Diagnosis of T2DM}

Diabetes is diagnosed based on the following stress-free hyperglycaemia levels [54] [55]:

- fasting plasma glucose (FPG) of $\geq 7.0 \mathrm{mmol} / \mathrm{L}(126 \mathrm{mg} / \mathrm{dL})$;

- plasma glucose of $\geq 11.1 \mathrm{mmol} / \mathrm{L}$ ( $200 \mathrm{mg} / \mathrm{dL}$ ) post-oral glucose tolerance test (OGTT), with $1.75 \mathrm{~g} / \mathrm{kg}$ ( $\max 75 \mathrm{~g}$ ) of anhydrous glucose;

- polyuria, polydipsia, nocturia, unexplained weight loss, or a random plasma glucose of $\geq 11.1 \mathrm{mmol} / \mathrm{L}(200 \mathrm{mg} / \mathrm{dL})$;

- glycated haemaglobin (HbA1c) of $>6.5 \%$.

The tests should follow the guidelines of the National Glycohemoglobin Stan- 
dardization Program (NGSP); results should be standardized to the Diabetes Control and Complications Trial (DCCT) assay. A HbAlc rate of $6.5 \%$ or less does not exclude diabetes diagnosed using glucose tests [55] [56]. Past epidemiological studies, which recommended usingHbA1c to diagnose diabetes, were only conducted on adult populations; thus, the independent use of $\mathrm{HbAlc}$ in diagnosing diabetes in children has not been validated. Future studies are needed to verify if adult $\mathrm{HbAlc}$-level standards may be used to diagnose diabetes in youth [44] [57].

\section{Screening for T2DM in Youth}

As with adults, T2DM is commonly undiagnosed in children. An effective screening of T2DM is necessary, since unrecognised hyperglycaemia will eventually cause life-threatening microvascular and macrovascular damage [40] [58]. According to the International Society of Pediatric and Adolescent Diabetes (ISPAD) 2014 guidelines, in most populations the screening of T2DM outside of research environments is not cost-effective [59]. A targeted screening remains the better option. The American Academy of Pediatrics, as well as the American Diabetes Association (ADA), suggest that diabetes screening begin once youth with high BMI ratings ( $>85$ percentile) reach ten years (or the onset of puberty, whichever is earlier), particularly those who are in high-risk groups: those who have a immediate family members with diabetes, are a member of high-risk racial/ethnic minorities, or display symptoms of insulin resistance such as polycystic ovarian syndrome (PCOS) and acanthosis nigricans [10]. For these children, testing should occur every two years, from the age of ten, by FPG or OGTT [10]. FPG is a more convenient and cost-effective diagnostic test, though OGTT tests and random postprandial blood glucose levels can confirm diabetes [35]. Results from studies using HbAlc to screen patients were inconsistent since one third of children with T2DM had normal HbA1c values [45].

\section{Clinical Treatment of T2DM in Youth with Drug-Therapy}

The American Diabetes Association (ADA) suggests that a diabetes response team should check-up on children with DM every 3 months [31]. This team should include nutritional, psychological, educational, and medical professionals who have experience with DM care. Treatment should aim to normalize blood glucose and HbA1c values. Successful control of the associated co-morbidities, such as hypertension and dyslipidemia, is also important [25]. The HbAlc levels should be assessed every three months during visits, with the final aim of reaching levels less than 7\%. Assessments of lipid levels, liver function, microalbuminuria levels, and sleep apnea symptoms should occur during annual visits [25] [31] [60].

Since studies on non-drug therapy are limited, traditionally, if the physiological goal of $\mathrm{HbAlc}<7 \%$ is not met with basic nutritional education and exercise, pharmacological therapy is employed [25] [61]. Many drugs are available for T2DM adult patients, although only metformin, a biguanide, is approved by the 
US Food and Drug Administration (FDA) as an oral medication for T2DM treatment in children [25]. The drug reduces hepatic gluconeogenesis and increases insulin-stimulated glucose uptake in fat and muscle, though it does not usually cause hypoglycaemia [46]. Metformin monotherapy is the optimal treatment for patients who are metabolically stable ( $\mathrm{HbAlc}<9 \%)$. The starting dosage is $500 \mathrm{mg}$ daily and, over four weeks, may increase to $1000 \mathrm{mg}$ twice daily [59]. Studies, with randomized controlled trials, suggest that Metformin is the most appropriate initial pharmacological treatment for youth with T2DM [62].

Insulin is prescribed for patients who are acutely ill or have significant hyperglycemia, ketonemia, ketonuria, polyuria, polydipsia, or weight loss. Insulin therapy is often reserved for patients who are unable to attain glycemic control with other drugs. Alternatively, insulin is used when other oral anti-diabetic drugs are harmful to patients (renal failure, hepatic failure, or heart failure) [10] [35]. The American Academy of Pediatrics Clinical Practice Guidelines suggests that insulin therapy be used for T2DM patients with a random blood glucose level greater than $250 \mathrm{mg} / \mathrm{dL}(13.9 \mathrm{mmol} / \mathrm{L})$ or a $\mathrm{HbAlc}$ level greater than $9 \%$ [60]. Many insulin regimens, such as basal insulin (0.25 - 0.5 units $/ \mathrm{kg}$ starting dose) or prandial insulin, effectively help patients attain standard metabolic levels [59].

Other therapies, although not FDA-approved for patients younger than 18 years, are still under investigation. The diabetes care team may use these drugs to improve glycemic control. Sulfonylureas (such as glyburide, glipizide, or glimepiride) elevate insulin secretion; thus, they require basal $\beta$-cell activity. Glucosidase inhibitors (such as acarbose and miglitol) inhibit intestinal uptake of carbohydrates. Incretins (such as exenatide) elevate postprandial insulin secretion. Thiazolidinediones (such as rosiglitazone and pioglitazone) raise insulin receptor sensitivity in muscle, adipose, and hepatic tissue [59] [63].

In extremely obese adolescents with T2DM, when lifestyle changes and pharmacotherapy have been unsuccessful, bariatric surgery may be required. Clinicians should aim to preserve $\beta$-cell secretory function [64]. The long-term outcomes of bariatric surgery in youth still need to be determined. Well-controlled studies of adolescent bariatric surgery are required to ascertain its full capability in obesity treatment.

\section{Prognosis of T2DM in Youth}

When treatment is ineffective, a longer duration of diabetes leads to a greater risk of complications, primarily causing injuries to blood vessels. Microvascular complications of T2DM include hypertension and hyperlipidemia states, which may lead to nephropathy, retinopathy, and neuropathy. Macrovascular risks include cardiovascular disease. Prompt diagnosis and therapy are required for preventing the disease's development. Studies suggest that complication risks occur earlier for youth diagnosed with T2DM than for patients who develop T2DM as adults [65]. Similarly, complication risks for youth with T2DM are higher than for similar-age youth with T1DM. The survival rate of youth-onset 
T2DM (YT2DM) is thus significantly worse than for T1DM. One study found that the renal survival rate for the T1DM group was $100 \%$ over 30 years, while the T2DM group survival rate was $100 \%$ at 10 years, $91.5 \%$ at 15 years, and only $77.5 \%$ at 20 years [66]. Using Markov modeling to predict the life-course of a hypothetical group with YT2DM (ages 15 - 24 years), researchers estimated a 15year loss of life-expectancy, as well as an average onset of chronic complications by the age of 40 [67]. Constantino et al. from Australia also reported that the survival rate was lower in the T2DM group than the T1DM group (hazard ratio 2.0 [95\% CI $1.2-3.2] ; \mathrm{p}=0.003$ ) with a cumulative mortality rate of $11 \%$ vs. $6.6 \%$. In particular, the rate of death because of cardiovascular malfunctions was higher for the T2DM group than the T1DM group (50\% vs. $30 \%, \mathrm{p}<0.035$; hazard ratio 3.5 [1.4 - 8.5]; $p=0.004$ ) [68]. In another study, Dart et al. claimed the survival percentage of T1DM vs. YT2DM after 10 years was $99.5 \%$ and $91.4 \%$, respectively, which dropped to $97.6 \%$ and $77.5 \%$ after 20 years [66]. This study further suggests that youth with T2DM suffer from greater complications than patients with other forms of diabetes mellitus. Another Canadian study of First Nation youth (likely to be T2DM) claimed a mortality rate after 25 years of $14.6 \%$, compared with a rate of $7.2 \%$ for non-First Nation youth-onset diabetes [69]. Studies of the mortality rates of T1DM patients compared with YT2DM patients are currently rare. The available data provides important comparative long-term mortality outcomes for YT2DM. They suggest a significantly higher mortality rate for youth with T2DM.

\section{Prevention of T2DM in Youth}

The financial and health consequences of the emerging T2DM epidemic call for an urgent public response. As drug therapies are costly, more emphasis should be placed upon preventive behaviours and early detection. Studies in China, Finland, and the USA have shown that T2DM rates are lowered by encouraging patients to shed between $7 \%-10 \%$ of body mass and to pursue moderate daily exercise [70]. Obesity, a warning signal of T2DM development, is the most prevalent clinical symptom in children with T2DM. Obesity results from genetic but also behavior-related factors, which include low amounts of physical exercise and heavy intakes of low-nutrient, high-sugar foods. Preventing childhood obesity aids efforts to lower T2DM rates. Small lifestyle changes, resulting in even a small amount of weight loss, may allow children and adolescents to avoid diabetes or delay its onset for many years [71]. By identifying children who are at higher risks for T2DM, clinicians may better offer services to prevent the development T2DM. Public health policies to lower obesity rates, which target large populations, are the most proactive treatment plan to date [10].

Prevention of T2DM in children and adolescents should start very early in life, perhaps even before birth, since the fetal and neonatal periods are crucial for organ development. Organs functionally develop from the fetal stage until childhood. Disturbance of the body's homeostasis during these crucial periods might increase the risk of insulin resistance, and even T2DM, in later life [72]. 
Many maternal factors during pregnancy augment the risk of children later developing diabetes, including diet and nutrition (malnutrition and overnutrition), health (T2DM and obesity), surrounding factors (biophenol A and hypoxia), hormone levels (testosterone and glucocorticoids), and even psychological stress [72]. The prevention of gestational DM may reduce the risk of T2DM subsequently developing in children [73]. Other factors that may raise the risk of offsprings developing T2DM include: late growth, early weaning, stress, and monosodium glutamate intake [72]. Indigenous people worldwide are disproportionately targets of diabetes. A study found that, in Manitoba, First Nations's women had a 3.5 fold risk, and their offspring had a 5 fold risk of developing diabetes compared to non-First Nations peers [74]. Low socioeconomic status and malnutrition may also increase risks of developing T2DM [72].

Developing preventive strategies on T2DM requires a multidisciplinary team. This team should help pregnant mothers maintain optimal blood glucose levels and receive nutritional diets. Studies suggest that positive results may be attained by combining good nutrition and moderate physical exercise for the mother, while closely observing the fetus's development in pregnant women [75]. Mothers should also avoid rapid increases in offspring weight [76]. Youth's dietary choices are easily affected by early instruction. A recent study in Manitoba suggests that since breastfeeding is correlated with a lower rate of diabetes among women and their children, it potentially decreases the risk of developing diabetes. Breastfeeding should thus be promoted in high-risk groups to help mitigate rates of both maternal and offspring diabetes [74].

The rise of childhood obesity is a key factor in the recent rise of T2DM in youth. Weight loss is thus a promising, though difficult, path of T2DM treatment. Health-care workers should help plan and initiate more school and community programs that persuade and educate families on undertaking more proactive attitudes towards healthy diets and daily physical exercise. Programs that encourage youth and their families to develop this positive will be effective in combating the ever-growing issue of obesity [10]. Results are greater when the entire family participates in healthy lifestyles. Family-based behavioral intervention plans are cost-effective in preventing T2DM, with results claiming significant weight loss sustained until ten years after treatment [77]. Examples of such family-based interventions for limiting obesity include nutritional education, increased opportunities for physical activity, reduced sedentary time, selfmonitoring of goals, stimulus control, and decreased caloric intake Successful healthier eating habits include drinking more water, reducing intake of sugarsweetened beverages (such as sodas, juices, and energy drinks), eating more fruits and vegetables, making healthy snack foods more visible in the kitchen, and limiting fast foods (such as high-fat, fried, large portioned meals) [77] [78] [79].

Besides healthy eating initiatives children lose excess weight when reducing sedimentary time to two hours daily (including TV, computer, and video games). To adopt fit lifestyles, youth should pursue 60 minutes of daily exercise, 
which could include walking or biking to school and work, dancing, taking strolls after meals, joining organized sports team, or even choosing stairs over elevators [59] [77] [78] [79]. There have been successful preventive programs in various countries. A study conducted with USA school-children found that a decrease in sedimentary time (from 12 to eight hours weekly) correlated with a decreased rate of body mass index (BMI) increase. In another lifestyle study in the UK, a decrease in student's consumption of sugared beverages led to significant losses of weight in overweight children [70].

Intervention is more effective at an early stage when blood glucose levels are still normal or elevated but not yet reflective of diabetes (impaired glucose tolerance (IGT) or impaired fasting glucose (IFG)). Recent observational studies have advocated that healthy lifestyles, which include healthy eating, higher levels of exercise, and moderate weight loss, help prevent the advancement of impaired glucose tolerance into diabetes mellitus. These lifestyles may help youth with T2DM better control glycemic levels [10] [80]. Effective plans of obesity prevention are particularly useful for ethnic minority youth, who have a disproportionate risk of developing obesity and T2DM. Studies show that ethnic minority families more frequently resign from obesity intervention programs [81]. Though T2DM patients often rely on drugs, studies have shown that Metformin use is not always effective at avoiding T2DM in obese youth [82]. Lifestyle intervention treatments, which have long-term benefits, are thus essential. These interventions, which promote weight management, nutritional education, and physical activity, should be available for all children at a high risk for the development of T2DM.

\section{Early Intervention and Non-Drug Therapy}

\subsection{Lifestyle Intervention}

Lifestyle interventions are an important preliminary treatment plan for adults with T2DM. Since many youth with T2DM are overweight, organized weightloss initiatives, such as healthier diets, moderate exercise, and reduced sedentary tendencies, help a wide range of patients. These activities all target to help youth reduce weight in the long term. Youth with T2DM are more likely to commit to lifestyle changes with the participation of their entire family. Most pediatric lifestyle intervention studies have focused on youth affected by obesity or pre-diabetes, instead of specifically on youth with T2DM. Yet since the conditions share many similarities, results may be applicable to both obesity and T2DM. In one comprehensive lifestyle intervention study, the Bright Bodies Weight Management Program for Children, which was a year-long lifestyle adjustment intervention for non-diabetic obese youth, results showed a decrease in BMI, cholesterol levels, and insulin resistance that lasted a year after intervention had finished [83]. Similarly, when pre-diabetic youth undergone the, Bright Bodies regimen for six months, participants had drastically lowered glucose and BMI $Z$ scores in comparison to control patients [84]. In another study, researchers tested the effects of lifestyle interventions with drug-therapy. They compared 
pre-diabetic groups (ages 10 - 17) given drugs (Metformin) and a high-carbohydrate diet or only a moderate-carbohydrate diet. After 6 months both groups, with no significant differences, had increased insulin sensitivity and lower BMI scores in comparison to control groups [85]. These findings support the intuitive theory that healthy lifestyles may supplement or replace drug-therapy and achieve physiologically measurable results. In another lifestyle study, researchers analyzed the link between rate of regular physical activity (RPA) on metabolic control and cardiovascular risk factors. Data was viewed from the Pediatric Quality Initiative (DPV), which synthesizes the records of 578 youth with T2DM (ages 10 - 20 years; mean $15.7 \pm 2.1$ years; $61.9 \%$ girls) from Germany and Austria.. Their results suggested that more frequent exercise correlated with decreased HbA1c levels, lower BMI ratings, and augmented HDL-cholesterol levels [80]. Other results supporting lifestyle interventions as effective treatments include a SEARCH for Diabetes in Youth study that placed 474 youth with T2DM into treatment groups of only lifestyle changes, Metformin, other oral hypoglycemic agents (OHA), insulin, and insulin with $\mathrm{OHA}(\mathrm{s})$. Participants treated with only lifestyle changes or Metformin had more favorable glucose levels than participants on other treatments [86]. These studies strongly suggest that, for obese patients, lifestyle interventions, optionally supplemented with medication, provide effective treatment.

For youth diagnosed with T2DM, evidence supporting lifestyle interventions is limited. Interventions are harder to succeed and often do not result in more favorable glucose levels [7]. One assessment of the overall treatments of 275 youth with T2DM (from 1995-2010) found that, after 12 months of lifestyle intervention, only $54 \%$ of 80 youth who initially had $\mathrm{HbAlc}$ levels of $<9.0 \%$ attained the target glycemic level of HbAlc $<7.0 \%$ [87]. In another study, the TODAY trial, which analyzed the intervention of 699 youth with T2DM (ages 10 - 17 years) assigned to treatments of either Metformin, Metformin with rosiglitazone, or Metformin with a family lifestyle intervention aimed at weight-loss. After an average of 3.9 years, results showed that $50 \%$ of the patients attained glycemic control with Metformin alone and Metformin with rosiglitazone. In contrast, after two years of lifestyle adjustments (200 - 300 minutes of weekly moderate exercise and 1200 - 1500 daily calorie consumption) with Metformin failed to attain optimal glycemic levels [88]. Contrasts in youth and adults with T2DM explain the insignificant effects that intensive lifestyle therapy had in youth with T2DM for attaining significant decreases in glycemic levels. Notably, the disease's pathophysiological progression $t$ is different for youths and adults. While in adults the onset and progress of hyperglycaemia takes years to form, both traits occur faster in youth [89]. The lack of progress may also be related to high rates of follow-up failures, factors relating to socioeconomic status, and high associated rates of depression. Interventions must balance the decrease in $\beta$-cell activity with an increase in insulin receptor sensitivity. While lifestyle intervention results in decreased BMI, this treatment had no effect on glycemic level regulations. More studies are needed to determine the level of weight loss 
required for improving glycemic control in youth. Involvement of an experienced dietitian is necessary in the integrated behavioral and medical team managing T2DM. This approach appears to offer the most effective treatment for optimal management [51] [88].

\subsection{Psychological Intervention}

While the unique physiological factors affecting youth with T2DM are important in treatment, psychosocial factors are equally important obstructions to lifestyle adjustments [90]. Studies on adults with T2DM indicate that poor moods elevate fasting glucose concentrations, suggesting a direct linkage between psychological health and physiological glucose levels. Therefore, psychologists may provide valuable care for youth with T2DM [24]. The WHO defines the social aspects of health as "the conditions in which people are born, grow, work, live, and age", which are "the fundamental drivers" of many health conditions. Socioeconomic status (SES), defined by income, schooling, and job, is a primary factor on the health of youth with T2DM [91]. Youth with T2DM are often from lower SES statuses and possess fewer social networks to help deal with the stressors in everyday life [24]. On a global scale, many high-risk populations for youth-onset T2DM have high rates of poverty and low education rates [92]. In the TODAY $(\mathrm{n}=699)$ group, $52.1 \%$ of patients lived in a single-parent household, $41.5 \%$ lived in households with an annual incomes fewer than $\$ 25,000$, and $26.3 \%$ had a parent with less than high-school schooling [51].

Recent reports indicate that obese youth, in comparison to healthy peers, suffer from elevated rates of depression [93]. The burden of neuropsychiatric disorders in young people with T2DM is high. In a study analyzing the effect of psychological barriers in youth with T2DM, Levitt Katz LE et al. shown that 20\% of participants had an existent psychological condition, such as depression, mood disorders, and schizophrenia [94]. Similarly, a SEARCH study claimed that youth with T2DM, especially boys, in comparison to those with T1DM, had a 3.48 fold risk of admitting depressed emotions. Overall, $20 \%$ of girls and 18\% of boys with T2DM admitted moderate to severe depression. These poor moods correlate with poor glycemic level regulation and increased emergency hospital trips. Youth with mildly depressed moods and males with moderately/severely depressed moods also had increased average HbAlc levels than males and females with normal moods [95]. Another study from TODAY claimed that 14.8\% of youth with T2DM expressed depressed moods, which was related to poorer qualities of life. Girls were also found to have higher occurrences of depression than boys. [96]. Another TODAY study stated that 678 youth-onset T2DM patients with clinical (6\%) and subclinical (20\%) rates of binge eating had drastically raised levels and rates of extreme obesity, depressive symptoms, and poorer quality of life [97]. Poor mental health and limited qualities of life help illuminate the difficulties patients face in adhering to lifestyle adjustments.

With mental health support, patients can better follow lifestyle adjustment regimens and achieve optimal glucose levels to avoid future complications. Suc- 
cessful interventions for youth with T2DM incorporates family members, considers cultural and family issues related to health behaviors, and accounts for competing priorities for resources. The most frequently employed theories are social cognitive theory (SCT), family systems theory (FST), and social ecological models (SEMs) [98]. Six types of psychological interventions were prioritized for inclusion into treatment: family therapy (including behavioral family systems therapy [BFST]), cognitive behavioral therapy (CBT), motivational interviewing, counseling, mentoring, and peer support (and peer-led interventions) [78].

Components of effective intervention for diabetic youth involve: 1) Coping skills training, which include group role-plays, diabetes management in social settings, and problem-solving skills training; 2) Internet self-care software, which include training in coping and participating in online social support groups; 3) Family teamwork, which include learning about developmentally appropriate self-management, developing plans for sharing responsibility, and training in communication skills; 4) Behavioral family systems therapy for diabetes, which include cognitive restructuring and conflict resolution; 5) Multisystemic therapy, which include exploring novel interventions of children's healthcare in multiple settings (e.g., home, community, school) that are tailored to individual and family needs (and which incorporate case management as needed); 6) Quality of life monitoring and feedback, which include using individually tailored motivational interviews and behaviorally informed communications as a part of routine care [98].

The growing body of behavioral intervention literature for youth with T1DM strongly supports a few, well-developed behavioral strategies that effectively promote well-being, adherence, and optimal glycemic controls [98]. Many studies demonstrate the effectiveness of these psychological interventions in improving outcomes for children and young people with T1DM [78]. Because of the lack of psychological research focused on youth with T2DM, few studies present data on mental health support in patients.

\section{Summary}

Type 1 diabetes mellitus (T1DM) remains the most common form of diabetes mellitus (DM) in childhood and adolescents. The prevalence of type 2 diabetes mellitus (T2DM) in the pediatric population around the world is rising rapidly because of the increasing prevalence of obesity, and minority groups are disproportionately affected. T2DM in children and adolescents is a serious medical problem that is more insidious than the adult-onset form and is more difficult to screen, diagnose, treat, and prevent. This diabetic condition in youth is also closely associated with high rates of complications. Treatment and prevention of T2DM should become important targets of public health programs. Currently, T2DM therapies rely heavily on drugs such as, Metformin and insulin (which are licensed for use in children under 18-year-olds), as the first-line treatment. Yet T2DM and obesity represent a significant public health issue that will, in the near future, pose a potentially great financial burden on governments, families 
and individuals; therefore, much more attention should be given to prevention and early intervention. These lifestyle interventions need to comprehensively address physical activity behaviors, family-based diets, diabetes education, and nutrition. A larger focus should be emphasized on the mental health and quality of life among children and adolescents with T2DM. There is little systematic data on psychosocial and behavioral interventions in children and adolescents with T2DM compared to T2DM in adults and T1DM in children and adolescents. This research is urgently needed.

\section{Competing Interests}

The authors declare that there are no competing interests regarding the publication of this paper.

\section{References}

[1] World Health Organization (2016) Global Report on Diabetes. World Health Organization, Geneva, 1-88.

[2] American Diabetes Association (2010) Diagnosis and Classification of Diabetes Mellitus. Diabetes Care, 33, S62-S69. https://doi.org/10.2337/dc10-S062

[3] Defronzo, R.A. (2009) Banting Lecture-From the Triumvirate to the Ominous Octet: A New Paradigm for the Treatment of Type 2 Diabetes Mellitus. Diabetes, 58, 773-795. https://doi.org/10.2337/db09-9028

[4] Arslanian, S. (2002) Type 2 Diabetes in Children: Clinical Aspects and Risk Factors. Hormone Research, 57, 19-28. https://doi.org/10.1159/000053308

[5] Vinicor, F. and Jack, L.J. (2004) 25 Years and Counting: Centers for Disease Control and Prevention Identifies Opportunities and Challenges for Diabetes Prevention and Control. Annals of Internal Medicine, 140, 943-944. https://doi.org/10.7326/0003-4819-140-11-200406010-00034

[6] Zeitler, P., Chou, H.S., Copeland, K.C. and Geffner, M. (2015) Clinical Trials in Youth-Onset Type 2 Diabetes: Needs, Barriers, and Options. Current Diabetes Report, 15, 1-8. https://doi.org/10.1007/s11892-015-0597-2

[7] Nadeau, K.J., Anderson, B.J., Berg, E.G., et al. (2016) Youth-Onset Type 2 Diabetes Consensus Report: Current Status, Challenges, and Priorities. Diabetes Care, 39, 1635-1642. https://doi.org/10.2337/dc16-1066

[8] May, A.L., Kuklina, E.V. and Yoon, P.W. (2012) Prevalence of Cardiovascular Disease Risk Factors among US Adolescents, 1999-2008. Pediatrics, 129, 1035-1041. https://doi.org/10.1542/peds.2011-1082

[9] Han, J.C., Lawlor, D.A. and Kimm, S.Y. (2010) Childhood Obesity. Lancet, 375, 1737-1748. https://doi.org/10.1016/S0140-6736(10)60171-7

[10] American Diabetes Association (2000) Type 2 Diabetes in Children and Adolescents. Diabetes Care, 23, 381-389. https://doi.org/10.2337/diacare.23.3.381

[11] Rhodes, E.T., Prosser, L.A., Hoerger, T.J., et al. (2012) Estimated Morbidity and Mortality in Adolescents and Young Adults Diagnosed with Type 2 Diabetes Mellitus. Diabetic Medicine, 29, 453-463. https://doi.org/10.1111/j.1464-5491.2011.03542.x

[12] Wild, S., Roglic, G., Green, A., et al. (2004) Global Prevalence of Diabetes. Diabetes Care, 27, 1047-1053. https://doi.org/10.2337/diacare.27.5.1047

[13] Dabelea, D., Bell, R.A., D’Agostino, R.B., et al. (2007) Incidence of Diabetes in 
Youth in the United States. JAMA, 297, 2716-2724.

https://doi.org/10.1001/jama.297.24.2716

[14] Fu, J., Liang, L., Gong, C., et al. (2013) Status and Trends of Diabetes in Chinese Children: Analysis of Data from 14 Medical Centers. World Journal of Pediatrics, 9 , 127-134. https://doi.org/10.1007/s12519-013-0414-4

[15] Schober, E., Holl, R.W., Grabert, M., et al. (2005) Diabetes Mellitus Type 2 in Childhood and Adolescence in Germany and Parts of Austria. European Journal of Pediatrics, 164, 705-707. https://doi.org/10.1007/s00431-005-1709-9

[16] Rotteveel, J., Belksma, E.J., Renders, C.M., et al. (2007) Type 2 Diabetes in Children in the Netherlands: The Need for Diagnostic Protocols. European Journal of Endocrinology, 157, 175-180. https://doi.org/10.1530/EJE-06-0754

[17] McMahon, S.K., Haynes, A., Ratnam, N., et al. (2004) Increase in Type 2 Diabetes in Children and Adolescents in Western Australia. The Medical Journal of Australia, 180, 459-461.

[18] Tajima, N. and Morimoto, A. (2012) Epidemiology of Childhood Diabetes Mellitus in Japan. Pediatric Endocrinology Reviews, 10, 44-50.

[19] Dean, H. (1998) NIDDM-Y in First Nation Children in Canada. Clinical Pediatrics, 37, 89-96. https://doi.org/10.1177/000992289803700205

[20] Dabelea, D., Hanson, R.L., Bennett, P.H., et al. (1998) Increasing Prevalence of Type II Diabetes in American Indian Children. Diabetologia, 41, 904-910. https://doi.org/10.1007/s001250051006

[21] Dabelea, D., Mayer-Davis, E.J., Saydah, S., et al. (2014) Search for Diabetes in Youth Study. Prevalence of Type 1 and Type 2 Diabetes among Children and Adolescents from 2001 to 2009. JAMA, 311, 1778-1786. https://doi.org/10.1001/jama.2014.3201

[22] Ke, C., Sohal, P., Qian, H., Quan, H., et al. (2015) Epidemiology Diabetes in the Young: A Population-Based Study of South Asian, Chinese and White People. Diabetic Medicine, 32, 487-496. https://doi.org/10.1111/dme.12657

[23] Liese, A.D., D’Agostino, R.B.J., Hamman, R.F., et al. (2006) Search for Diabetes in Youth Study Group. The Burden of Diabetes Mellitus among US Youth: Prevalence Estimates from the Search for Diabetes in Youth Study. Pediatrics, 118, 1510-1518. https://doi.org/10.1542/peds.2006-0690

[24] Sabin, M.A. (2013) Type 2 Diabetes in Children. Clinical Obesity, 3, 112-116. https://doi.org/10.1111/cob.12020

[25] Rosenbloom, A.L., Silverstein, J.H., Amemiya, S., et al. (2008) ISPAD Clinical Practice Consensus Guidelines 2006-2007. Type 2 Diabetes Mellitus in the Child and Adolescent. Pediatric Diabetes, 9, 512-526. https://doi.org/10.1111/j.1399-5448.2008.00429.x

[26] Pettitt, D.J., Forman, M.R., Hanson, R.L., et al. (1997) Breastfeeding and Incidence of Non-Insulin-Dependent Diabetes Mellitus in Pima Indians. Lancet, 350, 166-168. https://doi.org/10.1016/S0140-6736(96)12103-6

[27] Delva, J., O’Malley, P.M. and Johnston, L.D. (2006) Racial/Ethnic and Socioeconomic Status Differences in Overweight and Health-Related Behaviors among American Students: National Trends 1986-2003. Journal of Adolescent Health, 39, 536-545. https://doi.org/10.1016/j.jadohealth.2006.02.013

[28] Tamasan, I., Velea, I., Paul, C., et al. (2010) Type 2 Diabetes in Children and Adolescents-A Reality in Pediatric Pathology. Romanian Journal of Pediatrics, 59, 278283.

[29] Xue, Y., Gao, M. and Gao, Y. (2016) Childhood Type 2 Diabetes: Risk and Complications. Experimental and Therapeutic Medicine, 12, 2367-2370. 
[30] Badaru, A. and Pihoker, C. (2012) Type 2 Diabetes in Childhood: Clinical Characteristics and Role of B-Cell Autoimmunity. Current Diabetes Report, 12, 75-81. https://doi.org/10.1007/s11892-011-0247-2

[31] American Diabetes Association (2012) Diagnosis and Classification of Diabetes Mellitus. Diabetes Care, 35, S11-S71. https://doi.org/10.2337/dc12-s064

[32] Gunderson, E.P. (2008) Breast-Feeding and Diabetes: Long-Term Impact on Mothers and Their Infants. Current Diabetes Report, 8, 279-286. https://doi.org/10.1007/s11892-008-0050-x

[33] Ng, M.C., Shriner, D., Chen, B.H., et al. (2014) Meta-Analysis of Genome-Wide Association Studies in African Americans Provides Insights into the Genetic Architecture of Type 2 Diabetes. PLos Genetics, 10, e1004517. https://doi.org/10.1371/journal.pgen.1004517

[34] Kahn, S.E. (2003) The Relative Contributions of Insulin Resistance and Beta-Cell Dysfunction to the Pathophysiology of Type 2 Diabetes. Diabetologia, 46, 3-19. https://doi.org/10.1007/s00125-002-1009-0

[35] Vivian, E.M. (2006) Type 2 Diabetes in Children and Adolescents-The Next Epidemic? Current Medical Research and Opinion, 22, 297-306. https://doi.org/10.1185/030079906X80495

[36] Kiess, W., Böttner, A., Raile, K., et al. (2003) Type 2 Diabetes Mellitus in Children and Adolescents: A Review from a European Perspective. Hormone Research, 59, 77-84. https://doi.org/10.1159/000067829

[37] Roth, C.L. and Reinehr, T. (2010) Roles of Gastrointestinal and Adipose Tissue Peptides in Childhood Obesity and Changes after Weight Loss Due to Lifestyle Intervention. Archives of Pediatrics and Adolescent Medicine, 164, 131-138. https://doi.org/10.1001/archpediatrics.2009.265

[38] Welsh, J.A., Sharma, A.J., Grellinger, L., et al. (2011) Consumption of Added Sugars Is Decreasing in the United States. The American Journal of Clinical Nutrition, 94, 726-734. https://doi.org/10.3945/ajcn.111.018366

[39] Chen, L., Appel, L.J., Loria, C., et al. (2009) Reduction in Consumption of SugarSweetened Beverages Is Associated with Weight Loss: The Premier Trial. The American Journal of Clinical Nutrition, 89, 1299-1306. https://doi.org/10.3945/ajen.2008.27240

[40] Arslanian, S.A. (2000) Type 2 Diabetes Mellitus in Children: Pathophysiology and Risk Factors. Journal of Pediatric Endocrinology and Metabolism, 13, 1385-1394. https://doi.org/10.1515/jpem-2000-s612

[41] Hannon, T.S., Janosky, J. and Arslanian, S.A. (2006) Longitudinal Study of Physiologic Insulin Resistance and Metabolic Changes of Puberty. Pediatric Research, 60, 759-763. https://doi.org/10.1203/01.pdr.0000246097.73031.27

[42] Pinhas-Hamiel, O., Lerner-Geva, L., Copperman, N.M., et al. (2007) Lipid and Insulin Levels in Obese Children: Changes with Age and Puberty. Obesity, 15, 2825 2831. https://doi.org/10.1038/oby.2007.335

[43] Uwaifo, G.I., Fallon, E.M., Chin, J., et al. (2002) Indices of Insulin Action, Disposal, and Secretion Derived from Fasting Samples and Clamps in Normal Glucose-Tolerant Black and White Children. Diabetes Care, 25, 2081-2087. https://doi.org/10.2337/diacare.25.11.2081

[44] American Diabetes Association (2016) Classification and Diagnosis of Diabetes. Diabetes Care, 39, S13-S22. https://doi.org/10.2337/dc16-S005

[45] Reinehr, T. (2005) Clinical Presentation of Type 2 Diabetes Mellitus in Children and Adolescents. International Journal of Obesity, 29, S105-S110. 
https://doi.org/10.1038/sj.ijo.0803065

[46] Sarwar, N., Gao, P., Seshasai, S.R., et al. (2010) Diabetes Mellitus, Fasting Blood Glucose Concentration, and Risk of Vascular Disease: A Collaborative Meta-Analysis of 102 Prospective Studies. Lancet, 375, 2215-2222.

https://doi.org/10.1016/S0140-6736(10)60484-9

[47] O’Gara, P.T., Kushner, F.G., Ascheim, D.D., et al. (2013) 2013 ACCF/AHA Guideline for the Management of ST-Elevation Myocardial Infarction: A Report of the American College of Cardiology Foundation/American Heart Association Task Force on Practice Guidelines. Circulation, 127, e362-e425. https://doi.org/10.1161/CIR.0b013e3182742c84

[48] World Health Organization (2014) Diabetes Programme. World Health Organization, Geneva.

[49] Hillier, T.A. and Pedula, K.L. (2003) Complications in Young Adults with EarlyOnset Type 2 Diabetes: Losing the Relative Protection of Youth. Diabetes Care, 26, 2999-3005. https://doi.org/10.2337/diacare.26.11.2999

[50] Eppens, M.C., Craig, M.E. and Cusumano, J. (2006) Prevalence of Diabetes Complications in Adolescents with Type 2 Compared with Type 1 Diabetes. Diabetes Care, 29, 1300-1306. https://doi.org/10.2337/dc05-2470

[51] Copeland, K.C., Zeitler, P., Geffne, M., et al. (2011) Characteristics of Adolescents and Youth with Recent-Onset Type 2 Diabetes: The Today Cohort at Baseline. Journal of Clinical Endocrinology \& Metabolism, 96, 159-167. https://doi.org/10.1210/jc.2010-1642

[52] Rodriguez, B.L., Fujimoto, W.Y., Mayer-Davis, E.J., et al. (2006) Prevalence of Cardiovascular Disease Risk Factors in U.S. Children and Adolescents with Diabetes: The Search for Diabetes in Youth Study. Diabetes Care, 29, 1891-1896. https://doi.org/10.2337/dc06-0310

[53] Sellers, E.A.C., Yung, G. and Dean, H.J. (2007) Dyslipidemia and Other Cardiovascular Risk Factors in a Canadian First Nation Pediatric Population with Type 2 Diabetes Mellitus. Pediatric Diabetes, 8, 384-390. https://doi.org/10.1111/j.1399-5448.2007.00284.x

[54] Craig, M.E., Jefferies, C., Dabelea, D., et al. (2014) Definition, Epidemiology, and Classification of Diabetes in Children and Adolescents. Pediatric Diabetes, 15, 4-17. https://doi.org/10.1111/pedi.12186

[55] American Diabetes Association (2014) Executive Summary: Standards of Medical Care in Diabetes-2014. Diabetes Care, 37, S5-S13. https://doi.org/10.2337/dc14-S005

[56] Kapadia, C.R. (2013) Are the ADA Hemoglobin (A1C) Criteria Relevant for the Diagnosis of Type 2 Diabetes in Youth? Current Diabetes Report, 13, 51-55. https://doi.org/10.1007/s11892-012-0343-y

[57] Nowicka, P., Santoro, N., Liu,H., et al. (2011) Utility of Hemoglobin A1C for Diagnosing Prediabetes and Diabetes in Obese Children and Adolescents. Diabetes Care, 34, 1306-1311. https://doi.org/10.2337/dc10-1984

[58] Lobstein, T. and Leach, R. (2004) Diabetes May Be Undetected in Many Children in the UK. BMJ, 328, 1261-1262. https://doi.org/10.1136/bmj.328.7450.1261-c

[59] Zeitler, P., Fu, J., Tandon, N., et al. (2014) Type 2 Diabetes in the Child and Adolescent. Pediatric Diabetes, 15, 26-46. https://doi.org/10.1111/pedi.12179

[60] Copeland, K.C., Silverstein, J., Moore, K.R., et al. (2013) American Academy of Pediatrics. Management of Newly Diagnosed Type 2 Diabetes Mellitus (T2DM) in Children and Adolescents. Pediatrics, 131, 364-382. 
https://doi.org/10.1542/peds.2012-3494

[61] Springer, S.C., Silverstein, J., Copeland, K., et al. (2013) Management of Type 2 Diabetes Mellitus in Children and Adolescents. Pediatrics, 131, e648-e664.

https://doi.org/10.1542/peds.2012-3496

[62] Jones, K.L., Arslanian, S., Peterokova, V.A., et al. (2002) Effect of Metformin in Pediatric Patients with Type 2 Diabetes: A Randomized Controlled Trial. Diabetes Care, 25, 89-94. https://doi.org/10.2337/diacare.25.1.89

[63] Klein, D.J., Battelino, T., Chatterjee, D.J., et al. (2014) Liraglutide's Safety, Tolerability, Pharmacokinetics, and Pharmacodynamics in Pediatric Type 2 Diabetes: A Randomized, Double-Blind, Placebo-Controlled Trial. Diabetes Technology \& Therapeutics, 16, 679-687. https://doi.org/10.1089/dia.2013.0366

[64] Shah, A.S., D’Alessio, D., Ford-Adams, M.E., et al. (2016) Bariatric Surgery: A Potential Treatment for Type 2 Diabetes in Youth. Diabetes Care, 39, 934-940. https://doi.org/10.2337/dc16-0067

[65] White, N.H. (2015) Long-Term Outcomes in Youth with Diabetes Mellitus. Pediatric Clinics of North America, 62, 889-909. https://doi.org/10.1016/j.pcl.2015.04.004

[66] Dart, A.B., Sellers, E.A., Martens, P.J., et al. (2012) High Burden of Kidney Disease in Youth-Onset Type 2 Diabetes. Diabetes Care, 35, 1265-1271. https://doi.org/10.2337/dc11-2312

[67] Wong, J., Constantino, M. and Yue, D.K. (2015) Morbidity and Mortality in YoungOnset Type 2 Diabetes in Comparison to Type 1 Diabetes: Where Are We Now? Current Diabetes Report, 15, 1-11. https://doi.org/10.1007/s11892-014-0566-1

[68] Constantino, M., Molyneax, I.L., Limacher-Gisler, F., et al. (2013) Long-Term Complications and Mortality in Young-Onset Diabetes. Type 2 Diabetes Is More Hazardous and Lethal than Type 1 Diabetes. Diabetes Care, 36, 3863-3869. https://doi.org/10.2337/dc12-2455

[69] Dyck, R.F., Jiang, Y. and Osgood, N.D. (2014) The Long-Term Risks of End Stage Renal Disease and Mortality among First Nations and Non-First Nations People with Youth-Onset Diabetes. Canadian Journal of Diabetes, 38, 237-243. https://doi.org/10.1016/j.jcjd.2014.03.005

[70] Kaufman, F. (2007) Preventing Type 2 Diabetes in Children-A Role for the Whole Community. Diabetes Voice, 52, 35-38.

[71] Reinehr, T. (2013) Lifestyle Intervention in Childhood Obesity: Changes and Challenges. Nature Reviews Endocrinology, 9, 607-614. https://doi.org/10.1038/nrendo.2013.149

[72] Jiang, X., Ma, H., Wang, Y., et al. (2013) Early Life Factors and Type 2 Diabetes Mellitus. Journal of Diabetes Research, 2013, 1-11. https://doi.org/10.1155/2013/485082

[73] Sellers, E.A.C., Dean, H.J., Shafer, L.A., et al. (2016) Exposure to Gestational Diabetes Mellitus: Impact on the Development of Early-Onset Type 2 Diabetes in Canadian First Nations and Non-First Nations Offspring. Diabetes Care, 39, 2240 2246. https://doi.org/10.2337/dc16-1148

[74] Martens, P.J., Shafer, L.A., Dean, H.J., et al. (2016) Breastfeeding Initiation Associated with Reduced Incidence of Diabetes in Mothers and Offspring. Obstetrics \& Gynecology, 128, 1095-1104. https://doi.org/10.1097/AOG.0000000000001689

[75] Hui, A.L., Sevenhuysen, G., Harvey, D., et al. (2014) Food Choice Decision-Making by Women with Gestational Diabetes. Canadian Journal of Diabetes, 38, 26-31. https://doi.org/10.1016/j.jcjd.2013.08.263

[76] Hui, A.L., Back, L., Ludwig, S., et al. (2014) Effects of Lifestyle Intervention on Die- 
tary Intake, Physical Activity Level, and Gestational Weight Gain in Pregnant Women with Different Pre-Pregnancy Body Mass Index in a Randomized Control Trial. BMC Pregnancy and Childbirth, 14, 1-9. https://doi.org/10.1186/1471-2393-14-331

[77] Pulgaron, E.R. and Delamater, A.M. (2014) Obesity and Type 2 Diabetes in Children: Epidemiology and Treatment. Current Diabetes Report, 14, 1-12. https://doi.org/10.1007/s11892-014-0508-y

[78] National Collaborating Centre for Women's and Children's Health (2015) Diabetes (Type 1 and Type 2) in Children and Young People: Diagnosis and Management. National Collaborating Centre for Women's and Children's Health, London, 285447.

[79] Smart, C.E., Annan, F., Bruno, L.P., et al. (2014) Nutritional Management in Children and Adolescents with Diabetes. Pediatric Diabetes, 15, 135-153. https://doi.org/10.1111/pedi.12175

[80] Herbst, A., Kapellen, T., Schober, E., et al. (2015) Impact of Regular Physical Activity on Blood Glucose Control and Cardiovascular Risk Factors in Adolescents with Type 2 Diabetes Mellitus-A Multicenter Study of 578 Patients from 225 Centres. Pediatric Diabetes, 16, 204-210. https://doi.org/10.1111/pedi.12144

[81] Niet, J., Timman, R., Jongejan, R.M., et al. (2011) Predictors of Participant Dropout at Various Stages of a Pediatric Lifestyle Program. Pediatrics, 127, e164-e170. https://doi.org/10.1542/peds.2010-0272

[82] Wiegand, S., l'Allemand, D., Hübel, H., et al. (2010) Metformin and Placebo Therapy Both Improve Weight Management and Fasting Insulin in Obese Insulin-Resistant Adolescents: A Prospective, Placebo-Controlled, Randomized Study. European Journal of Endocrinology, 163, 585-592. https://doi.org/10.1530/EJE-10-0570

[83] Savoye, M., Nowicka, P., Shaw, M., et al. (2011) Long-Term Results of an Obesity Program in an Ethnically Diverse Pediatric Population. Pediatrics, 127, 402-410. https://doi.org/10.1542/peds.2010-0697

[84] Savoye, M., Caprio, S., Dziura, J., et al. (2014) Reversal of Early Abnormalities in Glucose Metabolism in Obese Youth: Results of an Intensive Lifestyle Randomized Controlled Trial. Diabetes Care, 37, 317-324. https://doi.org/10.2337/dc13-1571

[85] Garnett, S.P., Gow, M., Ho, M., et al. (2013) Optimal Macronutrient Content of the Diet for Adolescents with Prediabetes: Resist a Randomized Control Trial. Journal of Clinical Endocrinology \& Metabolism, 98, 2116-2125. https://doi.org/10.1210/jc.2012-4251

[86] Badaru, A., Klingensmith, G.J., Dabelea, D., et al. (2014) Correlates of Treatment Patterns among Youth with Type 2 Diabetes. Diabetes Care, 37, 64-72.

https://doi.org/10.2337/dc13-1124

[87] Wittmeier, K.D., Wicklow, B.A., Sellers, E.A., et al. (2012) Success with Lifestyle Monotherapy in Youth with New-Onset Type 2 Diabetes. Paediatrics \& Child Health, 17, 129-132. https://doi.org/10.1093/pch/17.3.129

[88] The Today Study Group (2012) A Clinical Trial to Maintain Glycemic Control in Youth with Type 2 Diabetes. New England Journal of Medicine, 366, 2247-2256. https://doi.org/10.1056/NEJMoa1109333

[89] Narasimhan, S. and Weinstock, R.S. (2014) Youth-Onset Type 2 Diabetes Mellitus: Lessons Learned from the Today Study. Mayo Clinic Proceedings, 89, 806-816. https://doi.org/10.1016/j.mayocp.2014.01.009

[90] Bernstein, C.M., Stockwell, M.S., Gallagher, M.P., et al. (2013) Mental Health Issues 
in Adolescents and Young Adults with Type 1 Diabetes: Prevalence and Impact on Glycemic Control. Clinical Pediatrics, 52, 10-15. https://doi.org/10.1177/0009922812459950

[91] World Health Organization (2015) Social Determinants of Health. The Solid Facts. 2nd edition, World Health Organization, Geneva.

[92] Gracey, M. and King, M. (2009) Indigenous Health Part 1: Determinants and Disease Patterns. Lancet, 374, 65-75. https://doi.org/10.1016/S0140-6736(09)60914-4

[93] Vander Wal, J.S. and Mitchell, E.R. (2011) Psychological Complications of Pediatric Obesity. Pediatric Clinics of North America, 58, 1393-1401.

https://doi.org/10.1016/j.pcl.2011.09.008

[94] Levitt Katz, L.E., Swami, S., Abraham, M., et al. (2005) Neuropsychiatric Disorders at the Presentation of Type2 Diabetes Mellitus in Children. Pediatric Diabetes, 6, 84-99. https://doi.org/10.1111/j.1399-543X.2005.00105.X

[95] Lawrence, J.M., Standiford, D.A., Loots, B., et al. (2006) Prevalence and Correlates of Depressed Mood among Youth with Diabetes: The Search for Diabetes in Youth Study. Pediatrics, 117, 1348-1358. https://doi.org/10.1542/peds.2005-1398

[96] Anderson, B.J., Edelstein, S., Abramson, N.W., et al. (2011) Depressive Symptoms and Quality of Life in Adolescents with Type 2 Diabetes: Baseline Data from the Today Study. Diabetes Care, 34, 2205-2207. https://doi.org/10.2337/dc11-0431

[97] The Today Study Group (2011) Binge Eating, Mood, and Quality of Life in Youth with Type 2 Diabetes: Baseline Data from the Today Study. Diabetes Care, 34, 858860. https://doi.org/10.2337/dc10-1704

[98] Hilliard, M.E., Powell, P.W. and Anderson, B.J. (2016) Evidence-Based Behavioral Interventions to Promote Diabetes Management in Children, Adolescents, and Families. American Psychologist, 71, 590-601. https://doi.org/10.1037/a0040359

Submit or recommend next manuscript to SCIRP and we will provide best service for you:

Accepting pre-submission inquiries through Email, Facebook, LinkedIn, Twitter, etc. A wide selection of journals (inclusive of 9 subjects, more than 200 journals)

Providing 24-hour high-quality service

User-friendly online submission system

Fair and swift peer-review system

Efficient typesetting and proofreading procedure

Display of the result of downloads and visits, as well as the number of cited articles

Maximum dissemination of your research work

Submit your manuscript at: http://papersubmission.scirp.org/

Or contact jdm@scirp.org 